\title{
Assessment of Breast Doses in Mammography
} Screening

\author{
1 Abdulrauf Maimani, ${ }^{2}$ Abdulraheem Abdulrahman Kinsara, ${ }^{2}$ Badar AlTaayeb and ${ }^{2}$ Nurul Islam Molla \\ ${ }^{1}$ College of Medicine, King Abdulaziz University Hospital, ${ }^{2}$ King Abdulaziz University, Department of Nuclear Engineering, Jeddah, KSA
}

Correspondence Address : N I Molla, E-mail: nimolla1971@gmail.com

\begin{abstract}
Thermoluminescent dosimeters (TLD-100H) were in use for the assessment of average glandular dose to breast in mammography screening with GE machine, King Abdul Aziz University Hospital for $\mathbf{4 5} \mathbf{~ m m}$ breast compressed thickness, 26 kVp, $100 \mathrm{mAs}$ and Mo/Mo target/filter combination. Reader's calibration factor for the TLDs was generated with the same exposure parameters using RadCal mammo ionization chamber as reference. The calibration factor was reproduced within $\mathbf{3 \%}$ standard deviation. Several sets of selected TLD-100H placing in different configurations were exposed to $\mathrm{X}$-ray beam under identical exposure condition as it was used for generation of calibration factor. The entrance surface doses were calculated by using the measured net TL-response and the calibration factor. Thereafter, the average glandular doses (AGD) were calculated. The measured average entrance surface dose (AGD) at the reference position $(6 \mathrm{~cm}$ laterally centered from the chest edge wall) was $7.704 \mathrm{mGy}$ with less than $2 \%$ standard deviation. The calculated average glandular dose to the breast at the reference location has been found to be $1.429 \pm 0.037 \mathrm{mGy}$.
\end{abstract}

Key words: TL-dosimetry, mammography, breast screening, entrance surface dose and average glandular dose.

\section{INTRODUCTION}

There is a significant risk of radiation induced carcinogenesis associated with the X-ray examinations of the female breast, particularly to the radiosensitive glandular tissues in the breast. Diagnostic exposure for medical examinations are based on the clinical needs as well as justified for the sake of benefits of accurate diagnosis of possible disease conditions. In practice doses to a particular target organs varies depending on specific machine parameters and study technique involving frequent over exposure than clinical needs. As such, standardization of radiation dose is required for a number of typical diagnostic medical procedures. Mammography is one of the widely used procedures for breast screening of small malignant lesions in the female breast, resulting significant reduction of breast cancer mortality, particularly in older women (1-4). Breast cancer is considered as the second highest cause of cancer deaths among the female patients in many countries. Age is the most important single risk factor in the development of breast cancer. Breast cancer incidence increases dramatically as a function of patient age (5-7). Mean glandular measurements results on women of age group between 49-65 years demonstrated expected increase of dose $(1-6$ mGy $)$ with breast thickness varied from 2 to $11 \mathrm{~cm}(8)$.

Statistics in mammography indicates that the incidence of breast cancer per 100,000 women increased by approximately $4 \%$ during the 1980 s and leveled off to about 100 cases per 10,000 women in 1990s. The reported death rates from breast cancer also declined significantly between 1992 and 1996. Medical doctors consider the reduction in breast cancer death via earlier detection and more effective treatment (9). In 2001, World Health Organization (WHO) predicted more than 1.2 million new breast cancer cases globally, while in the USA, both the American Cancer Society and the National Cancer Institute estimated approximately 192,200 cases of invasive breast cancer. The national diagnostic reference level (NDRL) of $3.5 \mathrm{mGy}$ for $55 \mathrm{~mm}$ thick breasts has been proposed by UK study in mammography screening as an appropriate value, to identify systems giving unusually high doses, with 
$3.5 \%$ of systems exceeding this level $(10,11)$. The role of dosimetric methods and influence of exposure parameters on the establishment of reference doses in mammography has been extensively studied by Zoetelief et al (12). They concluded that TLD measurements with $45 \mathrm{~mm}$ PMMA phantom representing $50 \mathrm{~mm}$ compressed breast thickness are the most reasonable approach for AGD estimation. Estimation of Patient average glandular dose to breast in mammography coupling with quantitative consideration of variables that affect the dose are compressed breast thickness, tube potential, tube loading, target/filter combination, magnification and beam collimation. Among them having the greatest effect are breast thickness and magnification. As for example, $8 \mathrm{~cm}$ breast receives about four times the mean tissue dose of $3.5 \mathrm{~cm}$ breast (13). The incident air kerma during mammography procedures were assessed using standard breast phantom and patient AGD at different institutes of Brazil by Khoury et al (14). This method involves recording the patient exposure parameters; $\mathrm{kVp}, \mathrm{mAs}$, arget/filter combination, and the incident air kerma measured with ionization chamber. The results showed a wide variation in the measured air kerma among the institutes ( $8.5 \mathrm{mGy}$ to $18.2 \mathrm{mGy}$ ). The results of patients' AGD values ranged from $0.23 \mathrm{mGy}$ to 7.46 mGy per film and AGD values per women were found between 8.23 and $40.6 \mathrm{mGy}$ in private hospitals, and 1.5 to $18.8 \mathrm{mGy}$ in public hospitals. Bulling and Nicoll (15) surveyed breast dose for a screening program in New Zealand. They found that while mean glandular dose from individual exposures ranged from 0.7-8.5 mGy, the total mean glandular dose per patient ranged from 4 to $29 \mathrm{mGy}$ due to multiple films being taken. Thilander-Klang et al found that substantial dose reduction up to $50 \%$ could be made with correct choice of X-ray spectrum maintaining good image quality (16).

An early detection by using modern mammography systems with automatic beam quality selection and alternative target filter combinations, introduced about $15 \%$ reduction in average received dose and up to $50 \%$ reduction in received doses to large breasts, resulting decrease of mortality rate significantly (17, 18). The AGD to glandular tissues in female breast is considered as the best indicator for the estimation of potential cancer risk from mammographic screening $(19,20)$. A significant increase of AGD in the beast screening over the limit usually associates greater risk. AGD is derived from measurement of the entrance surface air kerma (ESAK), or in some literatures defined as entrance surface dose (ESD), half-value layer (HVL) of the X-ray beam and the breast compressed thickness. Assumption for conversion factor CDG50,Ki,PMMA ( $\mathrm{mGy} / \mathrm{mGy}$ ) is used to calculate the average glandular dose (AGD) in different breast thicknesses. The assumption is based on the breast of composition 50\% fat and 50\% glandular tissue (3). In the recent years, these conversion factors have been extended to a larger interval of breast thicknesses and to account for other breast compositions and X-ray spectra $(7,21)$. Individual patient doses to breast glandular tissues are not possible to measure directly in mammography breast screening. However, several types of dosimeters are in use for breast dosimetry without much interfering diagnostic image quality. Thermoluminescent dosimeters (TLD), placed on the entrance surface of the breast, is considered as one of the tools of practical methodology in breast dosimetry. The present work has been conducted to justify the use of TL-dosimetry for the assessment of AGD to the breast in mammography screening. The research works have been accomplished using the $\mathrm{X}$ ray machine dedicated for mammography screening in the Radiology Department, King Abdulaziz University Hospital, Jeddah.

\section{MATERIALS AND \\ METHODS Calibration}

All the experimental measurements were done using

GE Senograph 2000D Mammography unit in King 
Abdul Aziz University Hospital, Jeddah. The X-ray beam from $26 \mathrm{kVp}, 100 \mathrm{mAs}$ and $\mathrm{Mo} / \mathrm{Mo}$ target/filter combination has been used for TLD calibration in terms of air kerma free in air. Selection of TL-dosimeters involved several steps in the processes, such as sensitivity of the group of TLDs on background response, annealing at $2400 \mathrm{C}$ for ten minutes, cooling to room temperature, reading out TL background response of each dosimeter in a single session, irradiating the selected chips with a fixed quantity of radiation from $90 \mathrm{Sr} / 90 \mathrm{Y}$ source. The irradiated chips were stored in a shielded container for overnight and thereafter all the TLD were read out with HARSHAW 4500 TLD reader in one session. Groups of TLD-100H chips having response variation within $2.5 \%$ standard deviation were selected for calibration. The calibration of the selected TLD-100H followed two step procedures. The first step involved, recording the exposure parameters and irradiation conditions (tube potential, tube loading, target/filtration combination) and measurement of Air kerma at the entrance surface of the breast for a selected projection. One set of five unexposed TLD-100H dosimeters were placed over $45 \mathrm{~mm}$ mammo phantom (bolus) at laterally centered $60 \mathrm{~mm}$ from the chest edge wall. TL dosimeters were irradiated with tube potential of $26 \mathrm{kVp}$, tube loading $100 \mathrm{mAs}$ and $\mathrm{Mo} / \mathrm{Mo}$ target/filter combination, shown in figure 1 . The TLD response $(\mathrm{nC})$ of each dosimeter was readout using HARSHAW 4500 reader. The TL response was subjected to correction for background, obtained from the average response of control dosimeters, and for sensitivity of each dosimeter (Element Correction Co-efficient). The second step was to measure exposure with SSDL level RadCal mammo ionization chamber under identical exposure condition as it was used for irradiation of TLD dosimeters. Placement of ionization chamber has been shown in Figure 2.

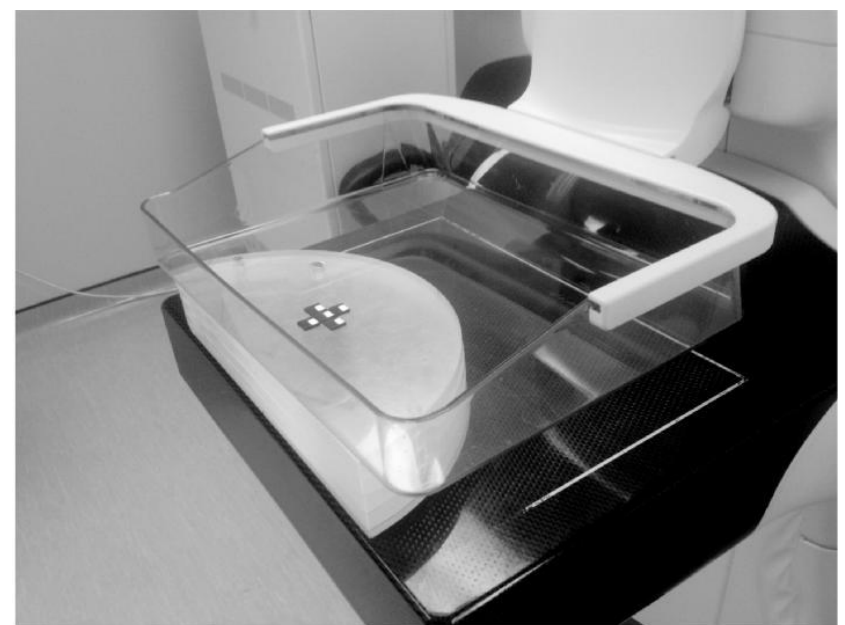

Figure 1. TLD set up for calibration

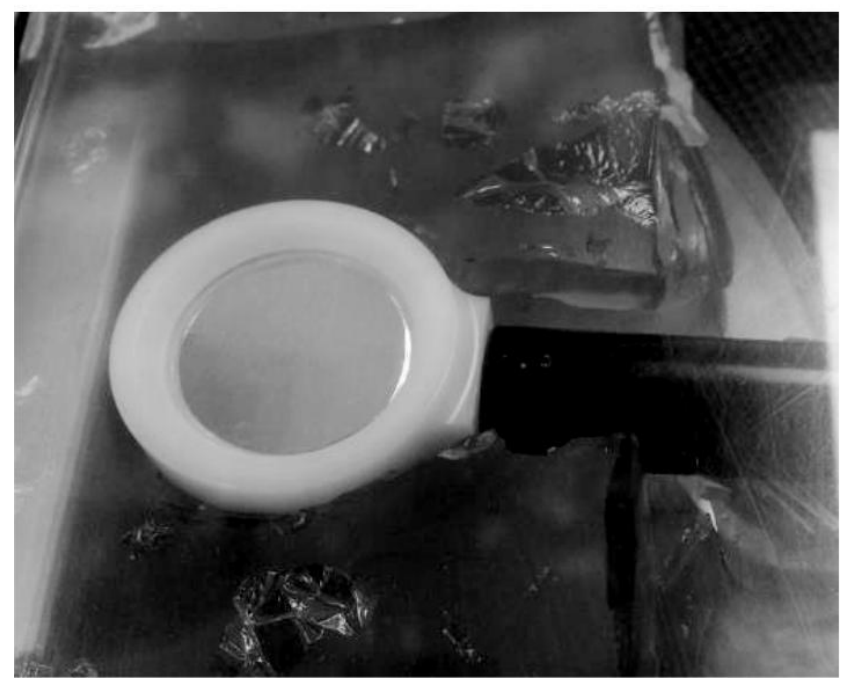

Figure 2. Placement of ionization for calibration experiment

The calibration factor has been generated using the following equation:

$\mathrm{CF}(\mathrm{nC} / \mathrm{mGy})=\mathrm{TLD}$ response $(\mathrm{nC}) /$ Ionization chamber reading (mGy) (i)

Where, $\mathrm{CF}$ is calibration factor for the measurement condition, $\mathrm{nC}$ is the average of net response (corrected for ECC) of TLDs, and Dose (mGy) is from ionization chamber readings.

\section{Average Glandular Dose (AGD)}

Four sets of unexposed new TLD-100H were used for the measurement of incident air kerma to the surface of breast target in order to calculate AGD to representative breast glandular tissues. The TL 
dosimeters were placed over $45 \mathrm{~mm}$ thick PMMA mammo phantom, representing $50 \mathrm{~mm}$ breast thickness, in different configurations. The TLDs placed at laterally centered position of $60 \mathrm{~mm}$ from the chest edge wall is considered as the reference location. They are shown in figure 3 (a, b, c, d). Each position over the phantom was grouped as the size of TLD before the insertions of the chips. Each set of TLDs was irradiated separately under identical exposure condition.

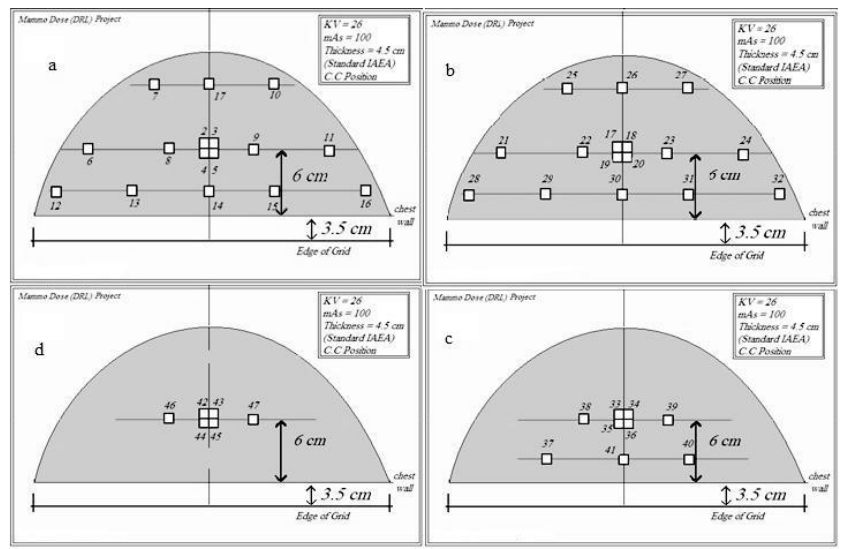

Figure 3. TLD placement during AGD measurement

Each set of TLDs was irradiated using tube potential (26 $\mathrm{kVp}$ ), tube loading ( $100 \mathrm{mAs}$ ) with $\mathrm{Mo} / \mathrm{Mo}$ target/filter combinations. Thereafter, TLDs were read out. AGD was calculated using the following equation.

$D_{G}=C_{\mathrm{DG} 50, \mathrm{Ki}, \mathrm{PMMA}} x s x K_{i} \quad \ldots$ (ii)

Where, DG is the dose to glandular tissue,

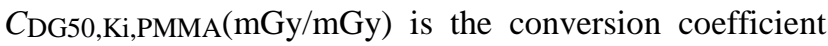
for the measured half value layer (HVL) and the

standard breast of $50 \mathrm{~mm}$ thickness with $50 \%$ glandularity (assumed) that is simulated by the 45 mm PMMA phantom, is spectral correction factor for the selected target/filter combination and $K_{i}$ is the incident air kerma. The incident air kerma has been calculated using the following equation:

$K_{i}=\frac{\text { Net Dose }(m G y)}{B}$

Where, ${ }^{\text {Net Dose }(m G y)}$ is the calculated value from TLD readings, and $\mathrm{B}$ is the backscatter correction factor for mammographic beam qualities as a function of HVL. The values of CDG50,Ki,PMMA, B and s were taken from reference (3). Wherever, necessary CDG50,Ki,PMMA ᄀand B value was interpolated.

\section{RESULTS}

The calculated calibration factor was $1208 \mathrm{nC} / \mathrm{mGy}$ with $\sim 3 \%$ standard deviation. The measured entrance surface dose to $45 \mathrm{~mm}$ breast compressed thickness with X-ray beam generated from $26 \mathrm{kVp}$, $100 \mathrm{mAs}$ and $\mathrm{Mo} / \mathrm{Mo}$ target/filter combination has been presented in Table 1. The entrance surface doses (ESD) at different position of breast phantom have been displayed graphically in Figures 4 -7.

Table 1. Measured values of entrance surface dose (ESD) using four sets of TLDs

\begin{tabular}{|c|c|c|c|c|c|c|c|}
\hline \multicolumn{2}{|c|}{$\begin{array}{l}\text { TLD setup } \\
\text { exp a }\end{array}$} & \multicolumn{2}{|c|}{$\begin{array}{c}\text { TLD setup } \\
\exp b\end{array}$} & \multicolumn{2}{|c|}{$\begin{array}{l}\text { TLD setup } \\
\text { exp c }\end{array}$} & \multicolumn{2}{|c|}{$\begin{array}{l}\text { TLD setur } \\
\text { exp d }\end{array}$} \\
\hline $\begin{array}{l}\text { TL } \\
\text { D } \\
\text { No. }\end{array}$ & $\begin{array}{l}\text { ESD } \\
\text { (mG } \\
\text { y) }\end{array}$ & $\begin{array}{l}\text { TLD } \\
\text { No. }\end{array}$ & $\begin{array}{l}\text { ESD } \\
\text { (mG } \\
\mathbf{y})\end{array}$ & $\begin{array}{l}\text { TL } \\
\text { D } \\
\text { No. }\end{array}$ & $\begin{array}{l}\text { ESD } \\
\text { (mG } \\
\mathbf{y})\end{array}$ & $\begin{array}{c}\text { TL } \\
\text { D } \\
\text { No. }\end{array}$ & $\begin{array}{l}\text { ESD } \\
\text { (mG } \\
\text { y) }\end{array}$ \\
\hline 1 & 7.52 & 17 & 7.73 & 33 & 7.73 & 42 & 7.73 \\
\hline 2 & 7.44 & 18 & 8.06 & 34 & 8.06 & 43 & 8.06 \\
\hline 3 & 7.52 & 19 & 8.11 & 35 & 8.11 & 44 & 8.11 \\
\hline 4 & 7.88 & 20 & 8.09 & 36 & 8.09 & 45 & 8.09 \\
\hline 5 & 7.26 & 21 & 8.31 & 37 & 8.31 & 46 & 8.31 \\
\hline 6 & 7.95 & 22 & 8.05 & 38 & 8.05 & 47 & 8.05 \\
\hline 7 & 8.21 & 23 & 8.13 & 39 & 8.13 & & \\
\hline 8 & 7.53 & 24 & 7.65 & 40 & 7.65 & & \\
\hline 9 & 7.26 & 25 & 7.26 & 41 & 7.26 & & \\
\hline 10 & 7.02 & 26 & 7.6 & & & & \\
\hline 11 & 7.51 & 27 & 7.59 & & & & \\
\hline 12 & 8.47 & 28 & 8.16 & & & & \\
\hline 13 & 8.65 & 29 & 8.04 & & & & \\
\hline 14 & 8.47 & 30 & 8.85 & & & & \\
\hline 15 & 8.37 & 31 & 7.98 & & & & \\
\hline 16 & 7.16 & 32 & 7.76 & & & & \\
\hline
\end{tabular}

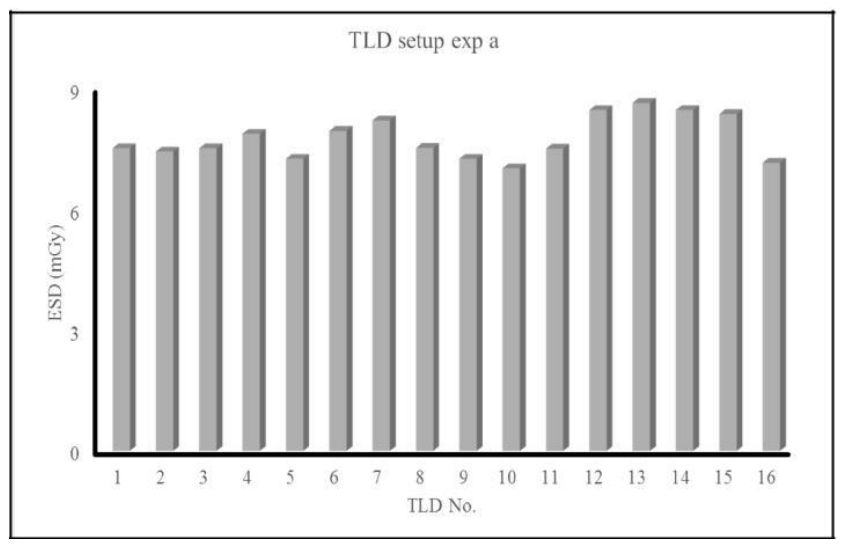

Figure 4. Entrance surface dose measured with experiment ' $a$ ' 


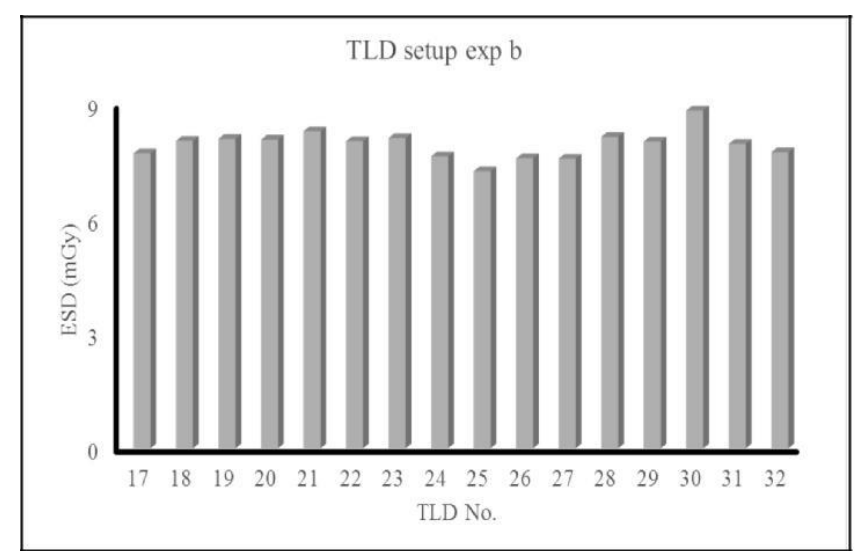

Figure 5. Entrance surface dose measured with experiment ' $b$ '

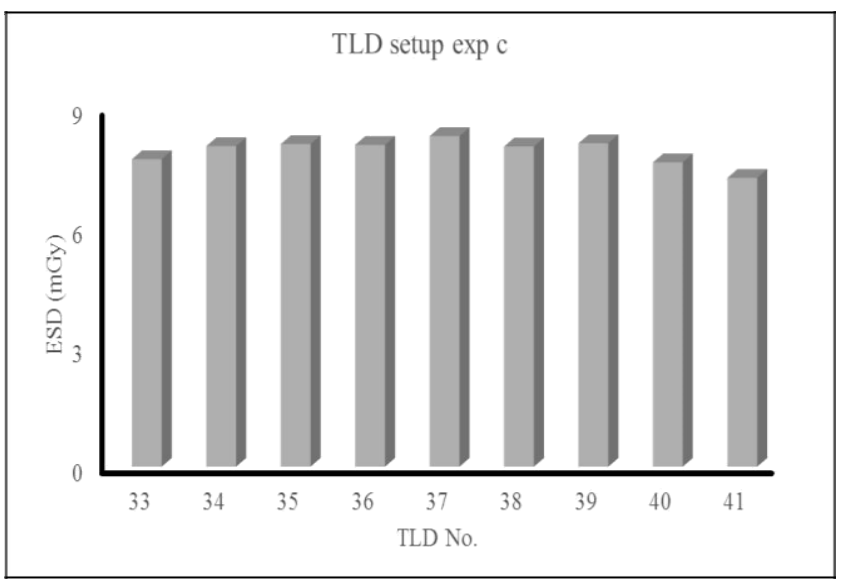

Figure 6. Entrance surface dose measured with experiment ' $c$ '

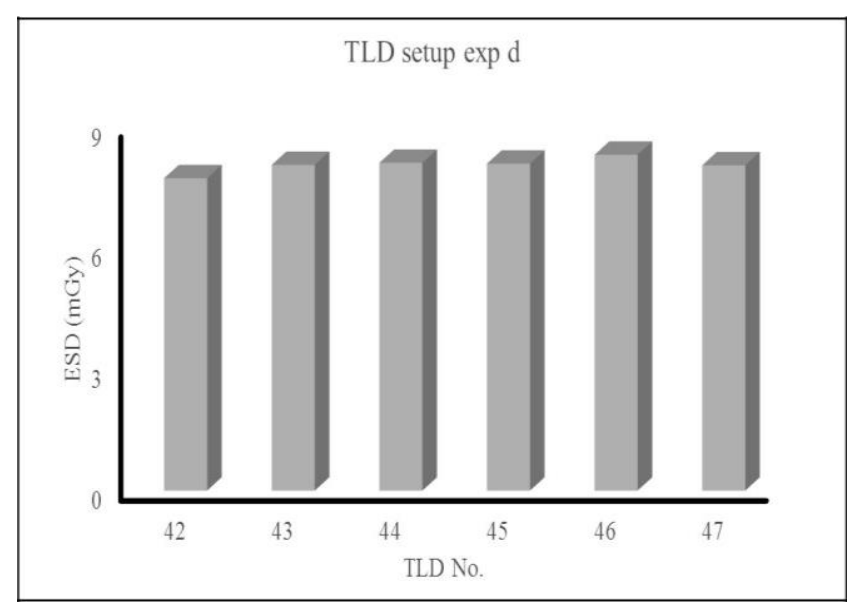

Figure 7. Entrance surface dose measured with experiment ' $d$ '

The Table 2 reads the calculated average glandular dose (AGD) corresponding to the use of different sets of dosimeters, placed with various configurations on the surface of the phantom. Figures 8-11 demonstrate the relative variation of AGD to the breast depending on the sites in the breast.
Table 2. The calculated values of average glandular dose (AGD) to the representative breast

\begin{tabular}{|c|c|c|c|c|c|c|c|}
\hline \multicolumn{2}{|c|}{ TLD sctup exp a } & \multicolumn{2}{|c|}{ TLD setup exp b } & \multicolumn{2}{|c|}{ TLD sctup exp c } & \multicolumn{2}{|c|}{ TLD sctup exp o } \\
\hline $\begin{array}{l}\text { TLD } \\
\text { No. }\end{array}$ & $\begin{array}{c}\text { AGD } \\
\text { (mGy) }\end{array}$ & TID No. & $\begin{array}{c}\mathbf{A G D} \\
(\mathrm{mG} \cdot \mathrm{y})\end{array}$ & $\begin{array}{c}\text { TLD } \\
\text { No. }\end{array}$ & $\begin{array}{c}\mathbf{A G D} \\
\text { (mGy) }\end{array}$ & $\begin{array}{c}\text { TLD } \\
\text { No. }\end{array}$ & $\begin{array}{c}\Lambda \text { GD } \\
\text { (mGy) }\end{array}$ \\
\hline 1 & 1.361 & 17 & 1.399 & 33 & 1.399 & 42 & 1.399 \\
\hline 2 & 1.347 & 18 & 1.459 & 34 & 1.459 & 43 & 1.459 \\
\hline 3 & 1.361 & 19 & 1.468 & 35 & 1.468 & 44 & 1.468 \\
\hline 4 & 1.426 & 20 & 1.464 & 36 & 1.464 & 45 & 1.464 \\
\hline 5 & 1.314 & 21 & 1.504 & 37 & 1.5114 & 46 & 1.504 \\
\hline 6 & 1.439 & 22 & 1.457 & 38 & 1.457 & 47 & 1.457 \\
\hline 7 & 1.486 & 23 & 1.472 & 39 & 1.472 & & \\
\hline 8 & 1.363 & 24 & 1.385 & 40 & 1.385 & & \\
\hline 9 & 1.314 & 25 & 1.314 & 41 & 1.314 & & \\
\hline 10 & 1.271 & 26 & 1.376 & & & & \\
\hline 11 & 1.359 & 27 & 1.374 & & & & \\
\hline 12 & 1.533 & 28 & 1.477 & & & & \\
\hline 13 & 1.566 & 29 & 1.455 & & & & \\
\hline 14 & 1.533 & 30 & 1.602 & & & & \\
\hline 15 & 1.515 & 31 & 1.445 & & & & \\
\hline 16 & 1.296 & 32 & 1.405 & & & & \\
\hline
\end{tabular}

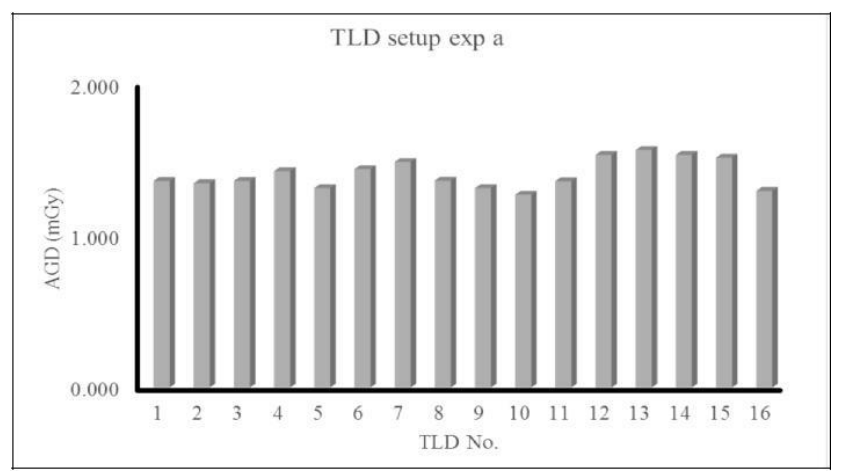

Figure 8. Variation of calculated average glandular dose to the breast

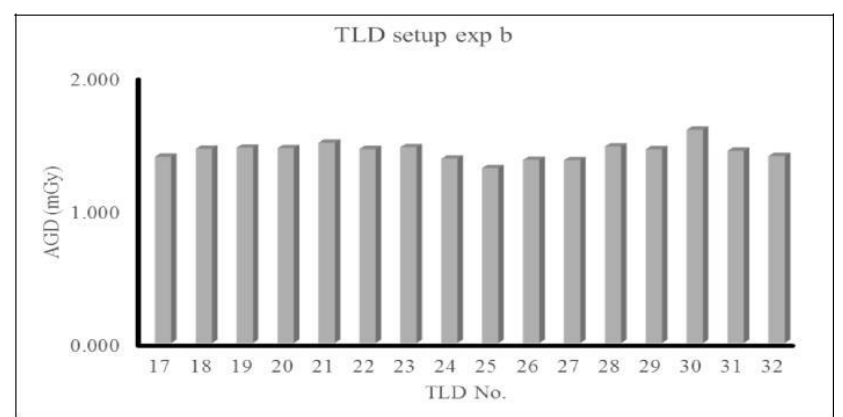

Figure 9. Variation of calculated average glandular dose to the breast

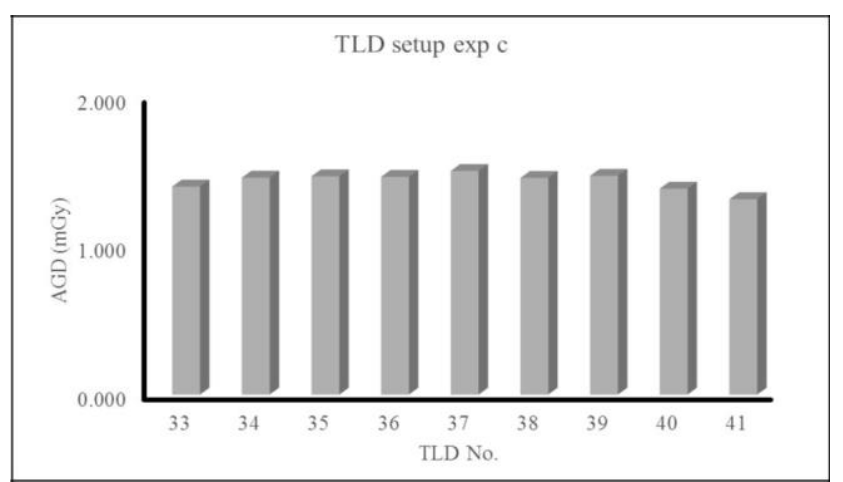

Figure 10. Variation of calculated average glandular dose to the breast 


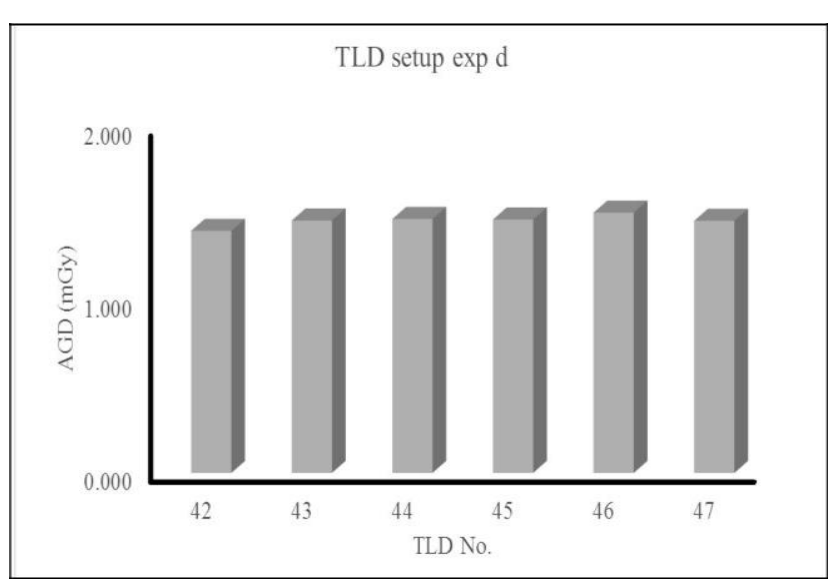

Figure 11. Variation of calculated average

\section{glandular dose to the breast DISCUSSION}

The set of four TLDs placed at the center (laterally 6 $\mathrm{cm}$ from chest wall edge) has been considered as the reference point. The average of the AGDs calculated from ESD values at this location is supposed to be the reference dose of this work. The quoted average glandular dose to the breast refers to the average of the AGD values, calculated by using the ESDs at this site with respect to four sets of TLDs. The average value of entrance surface dose (ESD) has been obtained as 7.704 mGy with less than $2 \%$ standard deviation. The corresponding average glandular dose has been calculated to be $1.429 \pm 0.037 \mathrm{mGy}$. There are other influence quantities such as back scatter factor, uncertainty in conversion coefficient, etc. Combining all the sources of errors in quadrature and the value of expanded uncertainty of measurement is between $8 \%$ and $14 \%$, considered as acceptable code of practice (3). Of them TLD measurements propagate an error up to $5 \%$. Zoetelief et al (12) recommended $1.6 \mathrm{mGy}$ AGD as the dose reference level for standard $45 \mathrm{~mm}$ PMMA phantom. D.R. Dance et al (6) quoted the average glandular dose of $1.2 \pm 0,5 \mathrm{mGy}$ for the range of breast thickness $50 \pm 3 \mathrm{~mm}$ from the work of Thilander et al (16). Assiamah et al (22) reported 1.45 $\pm 0.29 \mathrm{mGy}$ AGD value obtained from direct measurement of incident air kerma on $40 \mathrm{~mm}$ PMMA thickness using $26 \mathrm{kVp}$ beam. D. Bor et al (21) reported $1.34 \mathrm{mGy}$ and
$1.3 \mathrm{mGy}$ for $47.4 \mathrm{~mm}$ and $477 \mathrm{~mm}$ breast thickness, respectively using $27 \mathrm{kVp} \mathrm{Mo/Mo} \mathrm{target/filter} \mathrm{beam}$ quality. The AGD value of 1.429 mGy obtained in the present work fairly falls within the range compared to the results of the above mentioned data.

\section{CONCLUSION AND RECOMMENDATION}

TL-dosimetry appears to be a reliable technique for the quality assurance in the assessment of average glandular doses to the breasts in mammography screening. The research work reported here represent measurement with one particular mammography machine with a fixed set up of exposure parameters, and breast compressed thickness. The obtained AGD value not necessary represents the dose reference dose level (DRL) in mammography screening. However, the calculated AGD to representative breast thickness of 50 mm without compression obtained in this work may be considered as a baseline for future extension of this kind of research works at different mammography machine in other hospitals using both TLD and optically stimulated luminescence (OSL) nano Dot dosimeters.

\section{ACKNOWLEDGEMENT}

The authors express their gratitude and appreciation to the President, Vice President of Post Graduate Studies and Academic Research, and the Deanship of Scientific and Academic Research, King Abdulaziz University, for their financial and technical support under grant no. (017/428). The co-operation and vital support provided by Dr. M. Al-Johani (Chairman, Department of Nuclear Engineering, Director, Center for Training and Radiation Protection) is gratefully acknowledged. The research team is thankful to the staff members of mammography unit, Radiology Department, King Abdulaziz University Hospital for providing logistic support. Thanks to Mr. M. Yusuf, MS thesis student for his contribution in some computational works. Co-operation of Dr. M.H. Nassef and Dr. Sk. A. Latif is appreciated. 


\section{REFERENCES}

1. H. Y. Tsai, N. S. Chong, Y. J. Ho, and Y. S. Tyan, "Evaluation of Depth Dose and Glandular Dose For Digital Mammography". Radiation Measurements, vol. 45, pp. 72628,2010

2. I. Andersson, Mammography in Clinical Practice. Eastman Kodak Company, 1986.

3. IAEA, Dosimetry in Diagnostic Radiology: An International Code Practice, Technical Reports Series No. 457. Viena: IAEA, 2007

4. U. S. D. of Health, H. Services, and L. W. Bassett, "High Quality Mammography: Information for Referring Providers,” Breast J., vol. 1, no. 6, pp. 331-32, 1995

5. K.C. Young. "Radiation Doses in UK Trial of Breast Screening in Women Aged 40-48 years". The British J. of Radiology, 75, pp 362-70, 2002

6. D. R. Dance, C.L. Skinner, G. Alm Carlsson."Breast dosimetry". Appl. Radiat. and Isotopes, vol. 50, pp 185-203, 1999.

7. D. R. Dance, C. L. Skinner, K. C. Young, J. R. Beckett, and C. J. Kotre, "Additional Factors For the Estimation of Mean Glandular Breast Dose Using the UK Mammography Dosimetry Protocol," Phys. Med. Biol., vol. 45, no. 11, p. 3225, 2000.

8. K. C. Young, M. L. Ramsdale. Review of Dosimetric Methods for Mammography in the UK Breast Screening Program. Proceedings of a Training Workshop, Luxemburg, 23-25, 1997.

9. Thilander-Klang et al. Influence of Anode Filter Combinations on Image Quality and Radiation Dose in 965 Women Undergoing Mammography. Radiology, 203, 348-54, 1997.

10. European Protocol on Dosimetry in Mammography. European Commission Report EUR 16263, 1996.

11. K. C. Young, A. Burch and J. M. Oduko. Radiation Received in the UK Breast Screening Program in 2001-2002. The British Journal of Radiology, 78,207-18,2005.

12. J. Zoetelief, M. Fitzgerald, W. Leitz and M. Sabel. Dosimetric Methods For and Influence Of Exposure Parameters On the
Establishment Of Reference Doses In Mammography. Radia-tion Protection Dosimetry, 80(1-3),175-80,1998.

13. J. Law. Radiation Benefit and Risk at the Assessment Stage of the UK Breast Screening Program. British Journal of Radiolo-gy, 79, 479, 2006.

14. H. J. Khoury, V.S. Barros and C. Lopes. Evaluation of Patient Dose for Mammography in Pernambuco, Brazil. J. Epidemiol Community health, 55:204-12,2001.

15. S.M. Bulling and J. J. Nicoll. Level and Distribution of the Radiation Dose to the Population From a Mammography Screening Program in New Zealand. Radiation Protection dosimetry, 57, 455-58, 1995.

16. Thilander-Klang et al. Influence of Anode Filter Combinations On Image Quality and Radiation Dose in 965 Women Undergoing Mammography. Radiology, 203, 348-54, 1997.

17. S.R. Poliei, Jr. F.A.Mettler, S.A. Bartow, G. Maradian, M. Moskowitz. Occult Breast Cancer: Prevalence Andradiographic Detectability. Radiology, 163, p 459-62, 1987

18 S. Vinnicombe, S.M. Pinto Pereira, V.A. MeCormaek, S.Shiel, N. Perry, I.M. dos Santos Silva.Full-field Digital Versus Screen-Film Mammography: Comparison Within the U.K. Breast Screening Program Andsystematic Review of Published Data. Radiology, 251, p 347-58, 2009.

19. M.Chevalier, P. Moran, J.L. Ten, J.M. Fernandez Soto, T. Cepeda, E. Vano. Patient Dose in Digital Mammography. Med. Phys, 31, 2471-9,2004.

20. D. R. Dance, "Monte-Carlo Calculation of Conversion Factors for the Estimation of Mean Glandular Breast Dose," Phys. Med. Biol., vol. 35, no. 9, p. 1211, 1990.

21. D. Bor, S. Tukel, T. Olgar, T. Toklu, E. Aydın, and O. Akyol, "Investigation Of Mean Glandular Dose Versus Compressed Breast Thickness Relationship For Mammography," Radiat. Prot.Dosimetry, vol. 129, no. 1, pp. 160-64, 2008.

22. M. Assiamah, T.L. Nam, R.J. Keddy. Comparison of Mammography Radiation Dose Values Obtained From Direct Incident Air Kerma Measurements With Values From Measured X-ray Spectral Data. Appl. Radiat Isot., 62, pp 551-60, 2004. 